\title{
Cell-Penetrating HIV1 TAT Peptides Float on Model Lipid Bilayers ${ }^{\dagger}$
}

\author{
Corina Ciobanasu, ${ }^{*}$ Enno Harms, ${ }^{\star}$ Gisela Tünnemann, ${ }^{\S}$ M. Cristina Cardoso, ${ }^{\S, \|}$ and Ulrich Kubitscheck $* *$ \\ $\$$ Institute for Physical and Theoretical Chemistry, Rheinische Friedrich-Wilhelms-University Bonn, Wegelerstrasse 12, \\ D-53115 Bonn, Germany, ${ }^{\S}$ Max Delbrück Center for Molecular Medicine, Robert Rössle Strasse 10, D-13125 Berlin, Germany, and \\ "Department of Biology, Technische Universität Darmstadt, 64289 Darmstadt, Germany
}

Received March 4, 2009; Revised Manuscript Received April 28, 2009

\begin{abstract}
Cell-penetrating peptides like the cationic HIV1 TAT peptide are able to translocate across cell membranes and to carry molecular cargoes into the cellular interior. For most of these peptides, the biophysical mechanism of the membrane translocation is still quite unknown. We analyzed HIV1 TAT peptide binding and mobility within biological model membranes. To this end, we generated neutral and anionic giant unilamellar vesicles (GUVs) containing DPPC, DOPC, and cholesterol and containing DPPC, DOPC, cholesterol, and DPPS (DOPS), respectively. First, we characterized the mobility of fluorescently labeled lipids (TR-DHPE) within liquid-ordered and liquid-disordered lipid phases by single-molecule tracking, yielding a $D_{\mathrm{LO}}$ of $0.6 \pm 0.05 \mu \mathrm{m}^{2} / \mathrm{s}$ and a $D_{\mathrm{LD}}$ of $2.5 \pm 0.05 \mu \mathrm{m}^{2} / \mathrm{s}$, respectively, as a reference. Fluorescently labeled TAT peptides accumulated on neutral GUVs but bound very efficiently to anionic GUVs. Single-molecule tracking revealed that HIV1 TAT peptides move on neutral and anionic GUV surfaces with a $D_{\mathrm{N}, \mathrm{TAT}}$ of $5.3 \pm 0.2 \mu \mathrm{m}^{2} / \mathrm{s}$ and a $D_{\mathrm{A}, \mathrm{TAT}}$ of $3.3 \pm 0.2 \mu \mathrm{m}^{2} / \mathrm{s}$, respectively. TAT peptide diffusion was faster than fluorescent lipid diffusion, and also independent of the phase state of the membrane. We concluded that TAT peptides are not incorporated into but rather floating on lipid bilayers, but they immerged deeper into the headgroup domain of anionic lipids. The diffusion constants were not dependent on the TAT concentration ranging from $150 \mathrm{pM}$ to $2 \mu \mathrm{M}$, indicating that the peptides were not aggregated on the membrane and not forming any "carpet".
\end{abstract}

In the past decade, specific peptides and proteins have been shown to penetrate cell membranes while retaining their normal biological activity by a process called protein transduction $(1,2)$. The peptide sequences, which could be identified as being responsible for the translocation capability, were designated as protein transduction domains (PTDs) ${ }^{1}$ (3), cell-penetrating peptides (CPPs) (4), or Trojan horse peptides (5). Descriptions of the internalization process range from energy-independent cell penetration of membranes to endocytic uptake $(1,6-9)$. Certainly, the process varies for different types of PTDs. In general, however, the biophysical mechanism of the plasma

${ }^{\dagger}$ C.C. and U.K. gratefully acknowledge financial support by the European Union (EU-funded Marie Curie Research Training Network "BIOCONTROL"). U.K. and M.C.C. acknowledge financial support by the Volkswagen Foundation and the German Research Foundation.

*To whom correspondence should be addressed: Institute for Physical and Theoretical Chemistry, Wegelerstr. 12, D-53115 Bonn, Germany. Telephone: +49228 732262. Fax: +49228 739424.E-mail: u.kubitscheck@ uni-bonn.de.

${ }^{1}$ Abbreviations: CCD, charged coupled device; CPP, cell-penetrating peptide; DiI- $\mathrm{C}_{18}, 1,1^{\prime}$-dioctadecyl-3,3,3',3'-tetramethylindocarbocyanine; Bodipy-PC, 2-(4,4-difluoro-5,7-dimethyl-4-bora-3a,4a-diaza-s-indacene3-pentanoyl)-1-hexadecanoyl-sn-glycero-3-phosphocholine; DOPC, 1,2-dioleoyl-sn-glycero-3-phosphocholine; DPPC， 1,2-dipalmitoyl-sn-glycero3-phosphocholine; DPPS, 1,2-dipalmitoyl-sn-glycero-3-phospho-L-serine; DOPS, 1,2-dioleoyl-sn-glycero-3-phospho-L-serine; GUV, giant unilamellar vesicle; LSM, laser scanning microscope; PTD, protein transduction domain; TR-DHPE, Texas Red-labeled 1,2-dihexadecanoyl-sn-glycero-3-phosphoethanolamine. membrane translocation of the PTDs is not well understood. A highly charged cationic domain is common to all PTDs and essential for translocation. However, it is well-known that charged molecules cannot cross the lipid bilayer by passive diffusion due to the high Born charging energy encountered in a medium with a low dielectric constant. Cells usually utilize special transport systems such as ion carriers, channels, and ATP-coupled pumps to regulate the flow of ions and charged molecules across their membranes. From a physicalchemical point of view, it is therefore very surprising that short peptides containing a high percentage of cationic amino acids can nevertheless cross the plasma membrane of living cells by pathways that mostly appear to function without energy consumption.

One of the best-studied PTDs so far was derived from a polycationic protein, the transacting activator of transcription (TAT) of the human immunodeficiency virus (HIV-1). The so-called HIV1 TAT peptide corresponds to amino acids $48-57$ of the TAT protein and can translocate in bulk quantities across the plasma membrane of living cells within 3-5 min (10). Its cell membrane penetration capacity was exploited for the delivery of fluorophores, nucleotides, proteins, drugs, and genes into cells (see a review in ref 11 ). Proteins with a mass exceeding $100 \mathrm{kDa}, 40 \mathrm{~nm}$ nanoparticles, and even $200 \mathrm{~nm}$ liposomes have been delivered to the cellular interior using TAT peptides (12). 
The TAT PTD contains 10 amino acids comprising six arginine and two lysine residues, making the peptide highly cationic. It has been shown that substitution of a noncharged glutamine residue with alanine has no effect on cellular uptake, but substitution of any of the basic residues significantly decreases the rate of cellular uptake. The rate of cellular uptake also depends on the number of basic residues present, specifically, the number of arginine residues $(13,14)$. After the first claims of TAT PTD translocation across membranes in live cells in a so-called energy independent manner, more recent evidence has established that the intracellular entry of the TAT PTD does not occur at low temperatures, which was taken as an argument against passive membrane permeation (2). Probably, different endocytic pathways contribute to the internalization of CPP cargo systems: endocytosis (15), the caveolae-mediated endocytosis (16), or macropinocytosis (17). Meanwhile, it has been shown that TAT and other arginine-rich PTDs are internalized by means of all three endocytotic pathways (8). However, there are also experimental reports that clearly indicate that TAT peptides labeled with fluorophores can directly traverse cell membranes (10), and also model lipid bilayers of giant unilamelar vesicles (GUVs) but not large unilamellar vesicles of the same composition (18).

The classical models of how membrane-active antimicrobial peptides can cross biomembranes are the barrel-stave and the carpet model (19). In the barrel-stave model, a limited number of peptides first assembles on the lipid bilayer and then inserts into the bilayer, further peptides are then recruited, and a pore is formed. In the carpet model, peptides accumulate on the membrane surface until the integrity of the membrane is broken and pores are formed. At present, two mechanisms for how charged peptides could cross the hydrophobic barrier of a biological membrane without utilizing an active transport system are suggested (reviewed in ref 20). Complex formation of the cationic peptide with anionic cell surface components such as anionic lipid headgroups or the proteoglycan heparan sulfate, which is present on the surface of most cell plasma membranes $(21,22)$, could result in an electrically neutral complex, which could subsequently be taken up by adsorptive endocytosis $(23-25)$. An alternative hypothesis invokes the binding of PTDs to negatively charged lipids and disruption of the bilayer structure by formation of inverted micelles, resulting in their traversal into the cytoplasm (26).

In all models, the first step of the translocation is the association of a PTD peptide with components of the outer membrane leaflet. Although there were some early reports indicating the existence of specific cell surface receptors for the TAT protein $(27,28)$, experiments with $\mathrm{L}-$ and D-enantiomeric peptides $(14,29)$ do not support the idea of PTDs binding to specific receptors. It is generally accepted that electrostatic forces between the positively charged peptide TAT PTD and negative charges of phospholipids play a major role in the membrane binding of the peptide (30). In addition, nonelectrostatic forces, such as hydrogen bonding and hydrophobic or van der Waals forces, support binding of the TAT PTD to the membrane by contributing $\sim 20 \%$ to the binding energy (31). Recent results showed that the efficient binding of the TAT PTD requires a fluid membrane with lipids in the liquid-disordered state for the peptide-induced selective segregation of the negatively charged lipids, which is necessary to neutralize the positive charges on the peptide (30). However, it is unlikely that anionic lipids alone play a major part in the translocation of the TAT PTD across biological membranes. The binding of the TAT PTD to glycosaminoglycans on the outer cell surface is distinctly more probable for thermodynamic and statistical reasons (31).

A parameter, which directly would reflect possible aggregate formation preceding internalization, is the mobility of PTDs on the membrane surface. Aggregation and micelle formation should be clearly reflected in gross changes of the diffusion properties of the peptide within the membranes. These can most conveniently be studied by single-molecule observation using fluorescence microscopy employing electron multiplying CCD camera systems (32). Single fluorescently labeled molecules can be imaged microscopically as diffraction-limited spots. The intensity distribution of these spots may be approximated by a two-dimensional Gaussian function. Thus, the position of the molecule as the very center of the Gaussian can be determined with high precision by a fitting process. The localization precision may reach a few nanometers under optimal conditions (33). Thereby, fluorescence microscopy allows us to follow the traces of single molecules in time with a very high spatial precision. The technique has mostly been applied in analyzing the movement of single receptors and lipid molecules in biological membranes (reviewed in refs 34 and 35) but was recently extended to the study of single-molecule mobility within solution and the interior of mammalian cells (reviewed in refs 32 and 36 ).

In this study, we applied high-speed single-molecule tracking to the analysis of the interaction and mobility of fluorescently labeled TAT PTDs on the surface of neutral and anionic GUVs. As a reference, we first examined the mobility of fluorescent lipids within the GUV bilayer in liquid-ordered and liquid-disordered lipid phases. In all cases, we found that TAT peptides move on the biomembrane surface at a higher diffusion rate than the fluorescent lipid analogue. Lipid mobility varied clearly with the phase state of the membranes, whereas peptide mobility was independent of the membrane phase, suggesting that the TAT peptides were not incorporated into but rather floating on the membrane surface. Also, there was no indication of TAT peptide aggregation on the lipid bilayer for TAT concentrations of up to $2 \mu \mathrm{M}$.

\section{MATERIALS AND METHODS}

Lipids. The phospholipids 1,2-dipalmitoyl-sn-glycero-3-phosphocholine (DPPC), 1,2-dioleoyl-sn-glycero-3-phosphocholine (DOPC), 1,2-dipalmitoyl-sn-glycero-3-phospho-L-serine (DPPS), 1,2-dioleoyl-sn-glycero-3-phospho-L-serine (DOPS), and cholesterol were purchased from Sigma-Aldrich. The fluorescent probes Texas Red-labeled 1,2-dihexadecanoyl-sn-glycero-3phosphoethanolamine (TR-DHPE), 1,1'-dioctadecyl-3,3,3',3'tetramethylindocarbocyanine (DiI- $\mathrm{C}_{18}$ ), and 2-(4,4-difluoro5,7-dimethyl-4-bora-3a,4a-diaza-s-indacene-3-pentanoyl)-1-hexadecanoyl-sn-glycero-3-phosphocholine (Bodipy-PC) were obtained from Invitrogen $\mathrm{GmbH}$ (Karlsruhe, Germany) and Sigma-Aldrich (Schnelldorf, Germany).

Giant Unilamellar Vesicles. Giant unilamellar vesicles (GUVs) were created by electroformation according to refs 37 and 38. All phospholipids were dissolved in chloroform at a concentration of $1.27 \mathrm{mM}$. For creation of different types of vesicles, the following six lipid mixtures were prepared: DPPC and cholesterol at a molar ratio of 0.5:0.5 (designated as $\mathrm{S}_{\mathrm{N}}$ ); DOPC, DPPC, and cholesterol at a molar ratio of 0.33:0.33:0.33 $\left(\mathrm{M}_{\mathrm{N}}\right)$; 
Table 1: Composition of Giant Unilamellar Vesicles (GUVs) ${ }^{a}$

\begin{tabular}{llllll}
\hline & \multicolumn{5}{c}{ lipid molar ratio } \\
\cline { 2 - 6 } GUV & DPPC & DOPC & cholesterol & DPPS & DOPS \\
\hline $\mathrm{S}_{\mathrm{N}}$ & 0.5 & - & 0.5 & - & - \\
$\mathrm{M}_{\mathrm{N}}$ & 0.33 & 0.33 & 0.33 & - & - \\
$\mathrm{U}_{\mathrm{N}}$ & - & 0.5 & 0.5 & - & - \\
$\mathrm{S}_{\mathrm{A}}$ & 0.425 & - & 0.425 & 0.15 & - \\
$\mathrm{M}_{\mathrm{A}}$ & 0.283 & 0.283 & 0.283 & 0.15 & - \\
$\mathrm{U}_{\mathrm{A}}$ & - & 0.425 & 0.425 & - & 0.15 \\
\hline
\end{tabular}

${ }^{a}$ The designation of the respective lipid composition is given in the first column.

DOPC and cholesterol at a molar ratio of 0.5:0.5 $\left(\mathrm{U}_{\mathrm{N}}\right)$; DPPC, cholesterol, and DPPS at a molar ratio of 0.425:0.425:0.15 ( $\left.\mathrm{S}_{\mathrm{A}}\right)$; DOPC, DPPC, cholesterol, and DPPS at a molar ratio of 0.283:0.283:0.283:0.15 $\left(\mathrm{M}_{\mathrm{A}}\right)$; and DOPC, cholesterol, and DOPS at a molar ratio of $0.425: 0.425: 0.15\left(\mathrm{U}_{\mathrm{A}}\right)$. The lipid composition of the various GUVs was summarized in Table 1. Twenty microliters of the respective lipid mixture in chloroform were deposited on an indium tin oxide (ITO)-coated coverslip (from SPI Supplies, West Chester, PA), which was then dried in a desiccator under vacuum. The dried lipid film was covered with $235 \mu \mathrm{L}$ of $250 \mathrm{mM}$ sucrose in bidistilled water and covered with a second ITO coverslip. The latter was fixed at a distance of $2 \mathrm{~mm}$ from the first one, thus creating a chamber. For electroformation of GUVs, an alternating voltage of $1.2 \mathrm{~V} / 12 \mathrm{~Hz}$ provided by a pulse generator was applied for $2.5 \mathrm{~h}$ across the chamber. Then, the voltage was increased to $1.8 \mathrm{~V}$ for $30 \mathrm{~min}$ to detach the giant vesicles from the glass slides. The vesicles in the bulk volume were transferred into a $250 \mathrm{mM}$ glucose solution. The density difference between the outside (glucose) and the inside (sucrose) of the vesicles caused their sedimentation, allowing their optical analysis using an inverted light microscope. The electroformation of neutral and anionic GUVs (see Table 1 for lipid compositions of GUVs and the used nomenclature) in a transparent chamber was checked by differential interference contrast (DIC) microscopy, and also by confocal laser scanning microscopy. Addition of fluorescent lipid analogues DiI- $\mathrm{C}_{18}$ and Bodipy-PC at $0.1 \mathrm{~mol} \%$ to the lipid mixture allowed observation of the formation of vesicles by confocal laser scanning microscopy. As expected, confocal imaging of GUVs formed from the lipid mixtures $\mathrm{M}_{\mathrm{N}}$ and $\mathrm{M}_{\mathrm{A}}$ revealed a domain structure (39), because both Bodipy-PC and DiI- $\mathrm{C}_{18}$ preferentially localize in DOPC-rich liquid-disordered lipid domains (40) (see OSM Figure 1A of the Supporting Information).

Peptides. The biotinylated TAT peptide corresponding to amino acids $48-57$ of the HIV1 TAT protein (Biotin-AhxYGRKKRRQRRR; molecular mass of $1.87 \mathrm{kDa}$, abbreviated as b-TAT), the rhodamine-labeled, retro-all D TAT peptide (5,6-TAMRA-RRRQRRKKRG; molecular mass of $1.81 \mathrm{kDa}$, abbreviated as R-TAT), and the Alexa Fluor 647-labeled, retro-all D TAT peptide (Alexa Fluor 647-RRRQRRKKRG; molecular mass of $1.4 \mathrm{kDa}$, abbreviated AF-TAT) were purchased from Peptide Specialty Laboratories $\mathrm{GmbH}$ (Heidelberg, Germany).

Sample Preparation for Light Microscopy. For measurements focusing on the interaction of TAT with the GUVs, the glucose solution was mixed with the TAT peptides before addition of the GUVs. Fluorescent R-TAT was used at a concentration of $250 \mathrm{pM}$, or at a concentration of $100 \mathrm{pM}$ in a mixture with unlabeled b-TAT at a total concentration $2 \mu \mathrm{m}$. The sample chamber had a total volume of $400 \mu \mathrm{L}$, and the final molar ratio of lipids to TAT in the latter experiment was 27.5:1. In the experiments using $250 \mathrm{pM}$ TAT, the respective molar ratio was 220000:1. The incubation time of GUVs in the peptide/glucose solution before we began image acquisition was $30 \mathrm{~min}$.

Confocal Microscopy. GUV formation and TAT peptide binding to the GUVs were examined by a confocal laser scanning microscope (LSM 510 Meta from Carl Zeiss, Jena, Germany) equipped with a plan apochromat $63 \times$ objective lens at room temperature. DiI- $\mathrm{C}_{18}$ and R-TAT were excited with the $543 \mathrm{~nm}$ line of a He-Ne laser, while TR-DHPE was excited using a red $\mathrm{He}-\mathrm{Ne}$ laser emitting at $633 \mathrm{~nm}$. For fluorescence detection, standard filter settings for fluorescein and rhodamine were employed.

Single-Molecule Microscopy. Single-molecule experiments were performed to follow the single-molecule trajectories of lipid tracer molecules (DiI-C ${ }_{18}$ and TR-DHPE) and R-TAT peptides. The experiments were done at room temperature using an inverted wide-field single-molecule microscope based on a Axiovert 200TV (Zeiss, Jena, Germany) equipped with a $63 \times$ NA 1.2 water immersion objective lens and a 4-fold magnifier at the camera port. An iXon BI DV-860 camera (Andor Technologies, Belfast, Northern Ireland) with a pixel size of $24 \mu \mathrm{m}$ was used for image acquisition. For peptide analysis, a single-frame integration time of $10 \mathrm{~ms}$ at a frame rate of $100 \mathrm{~Hz}$ was used, whereas an integration time of $33 \mathrm{~ms}$ at a frame rate of $30 \mathrm{~Hz}$ was used for lipid imaging. Fluorescence was excited with laser light (irradiance of $0.5 \mathrm{~kW} / \mathrm{cm}^{2}$ ), which was coupled into the epi-illumination light path of the microscope by an optical monomode fiber (for details, see ref 32). All singlemolecule tracking experiments were performed on the top surface of freshly prepared GUVs. The adjustment of the correct focal plane was done as follows. First, the radius of the GUV in the equatorial plane was measured employing DIC and a grid with known dimensions within the eyepiece. Then, the focus was shifted into the sample by a distance corresponding to the GUV radius to reach the top pole of the spherical GUV. Only in this plane could single-molecule signals be observed using the fluorescence mode. Since the radius of the GUVs was in the range of $40 \mu \mathrm{m}$, the curvature of the surface was small enough to allow the imaging of an extended region in the top region of the GUV, because the axial resolution was $\sim 1 \mu \mathrm{m}$. The observable, circular region on the GUV top had a diameter of $\sim 14 \mu \mathrm{m}$. From this field, usually 1000-2000 single frames were recorded in a single movie. For each experimental situation, a total of three independent experiments comprising 15 movies were obtained, and the trajectory data were pooled.

Trajectory Analysis. Identification and tracking of the single-molecule signals were accomplished using Diatrack 3.02 (Semasopht, Chavannes, Switzerland), a commercial image processing program for the localization of single objects and construction of trajectories. All further data processing was performed with ImageJ (41) and Origin 8.0 (OriginLab Corp., Northampton, MA). Each single-molecule trajectory was defined by coordinates $\left(x_{i}, y_{i}\right)$. The running frame number was denoted by $i(1 \leq i \leq N$, where $N$ is the number of frames in which the molecule was observed). For each trajectory, the squared displacements $r^{2}\left(n t_{\mathrm{c}}\right)$ were computed with $t_{\mathrm{c}}$ representing the imaging cycle time (inverse frame rate) and $n$ the difference of the running index. Therefore, $n t_{\mathrm{c}}$ denoted the time between 
two observations of the same molecule. By averaging the square displacements for identical $n t_{\mathrm{c}}$ values, the mean square displacements (MSD), $\left\langle r^{2}\left(n t_{\mathrm{c}}\right)\right\rangle$, were obtained. For two-dimensional Brownian motion, the MSD is given by

$$
\left\langle r^{2}\left(n t_{\mathrm{c}}\right)\right\rangle=4 D\left(n t_{\mathrm{c}}\right)
$$

A linear relationship between MSD and time indicates free Brownian motion. In this way, diffusion coefficients can be derived from single-molecule trajectories, if they are long enough to generate significant mean values. Generally, the MSD values of many different trajectories were averaged for identical values of $n t_{\mathrm{c}}$ to improve statistics. However, a joint analysis of the trajectories according to eq 1 is not suitable, when the population contains different mobility fractions.

Jump Distance Analysis. The probability that a molecule starting at a given position will be found in a distance $r$ within a shell of width $\mathrm{d} r$ from the start position at time $t$ is given by (42)

$$
p(r, t) \mathrm{d} r=\frac{1}{4 \pi D t} \mathrm{e}^{-r^{2} / 4 D t} 2 \pi r \mathrm{~d} r
$$

Equation 2 is valid for a single particle species diffusing in two dimensions. Experimentally, this probability distribution can be approximated by a frequency distribution, determined by counting the jump distances within respective intervals $[r, r+\mathrm{d} r]$ traveled by single particles after $n t_{\mathrm{c}}$. When several particle subspecies with different mobilities are present, the jump distance distributions cannot be fitted by eq 2 with a single diffusion coefficient. However, such particle populations can be analyzed by curve fitting using more than one term according to eq 2. For a jump distance distribution containing contributions from two different subspecies

$$
p^{\prime}(r, t) \mathrm{d} r=\sum_{j=1}^{2} \frac{M f_{j}}{2 D_{j} t} \mathrm{e}^{-r^{2} / 4 D_{j} t} r \mathrm{~d} r
$$

where $M$ is the total number of jumps considered in the analysis and $f_{1}$ and $f_{2}$ designate the fractions with diffusion constants $D_{1}$ and $D_{2}$, respectively. In analyzed jump distributions, the distances between molecular positions in successive frames were evaluated.

\section{RESULTS}

Imaging and Tracking of Single-Lipid Analogues within Anionic and Neutral GUVs. To obtain insight into the dynamics of the chosen model bilayer systems, we performed measurements of lipid mobility in GUVs with different lipid compositions. Lipid diffusion in model membranes can be analyzed by imaging and tracking single, dye-coupled lipid analogue molecules $(43,44)$. We used fluorescent TR-DHPE as a tracer molecule. Six different sets of experiments were performed using liquid-ordered GUVs formed by DPPC and cholesterol $\left(\mathrm{S}_{\mathrm{N}}\right)$, liquid-disordered GUVs formed by DOPC and cholesterol $\left(\mathrm{U}_{\mathrm{N}}\right)$, and GUVs formed by equimolar ternary mixtures of DOPC, DPPC, and cholesterol $\left(\mathrm{M}_{\mathrm{N}}\right)$. In addition to these mixtures of neutral components, we examined anionic GUVs created by addition of $15 \mathrm{~mol} \%$ DOPS or DPPS (indicated by the subscript "A" instead of "N"). The cholesterol was added to better mimic mammalian cell membranes (45).
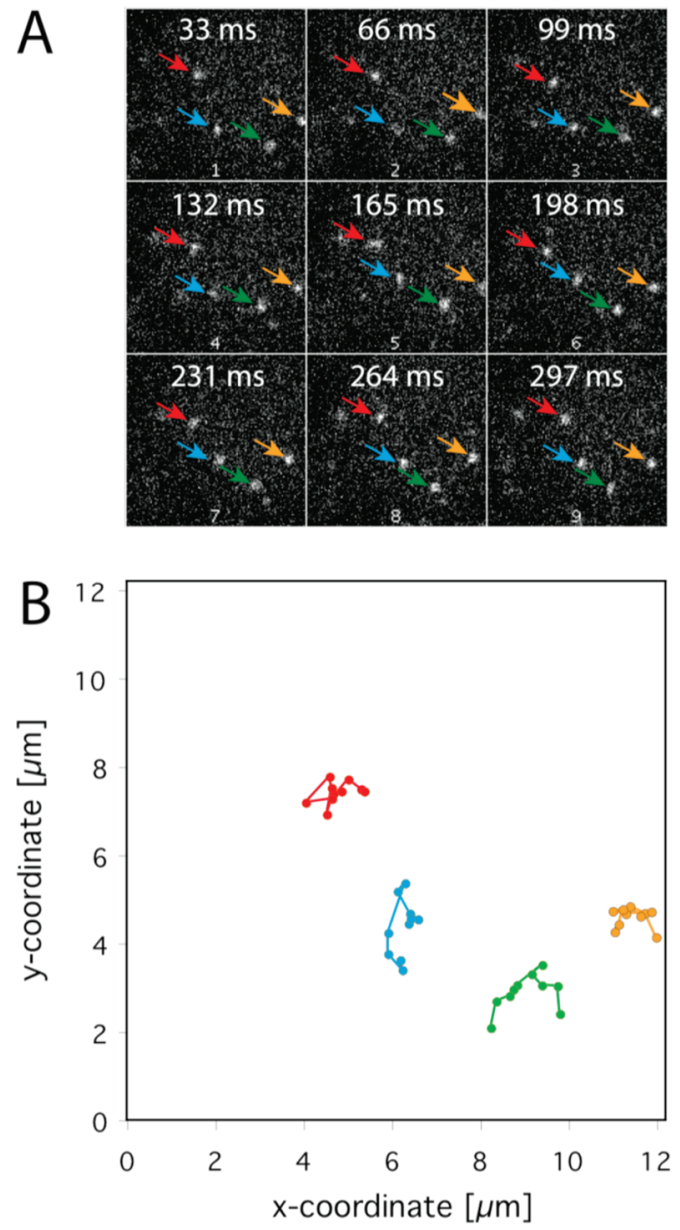

FIgURE 1: Single TR-DHPE molecules diffusing within the GUV membrane. (A) Nine subsequent movie frames illustrate the motions of four single TR-DHPE molecules (marked by arrows) in a total time of $300 \mathrm{~ms}$ in the two-component GUVs (DOPC, DPPC, and cholesterol at a molar ratio of 0.33:0.33:0.33). The tracer molecules were present in the lipid mixture at $10^{-7} \mathrm{~mol} \%$. The field of view of a single frame was $(12.2 \mu \mathrm{m})^{2}$ and contained the upper segment of a single GUV. (B) Trajectories of two-dimensional diffusion of the same four particles in GUV membranes as determined by the tracking software. The shown area corresponds to the microscopic field of view.

Also, it contributes to a better distribution of the domains in GUVs (40).

We imaged the tracer TR-DHPE within neutral GUVs (lipid mixtures $\mathrm{S}_{\mathrm{N}}, \mathrm{U}_{\mathrm{N}}$, and $\mathrm{M}_{\mathrm{N}}$ ). The tracer molecules were added in a ratio of $10^{-7} \mathrm{~mol} \%$ to the lipids before preparation of the GUVs. GUVs were created by electroformation and then transferred to the microscope sample chamber. Single diffraction-limited spots of fluorescence could be observed in the single-molecule microscope, when the focal plane was adjusted to the upper GUV surface. Acquisition of movies at a frame rate of $30 \mathrm{~Hz}$ revealed that these fluorescence spots were rapidly moving in the GUV surface (see movie S1 of the Supporting Information). A sequence of successive movie frames is shown in Figure 1, which illustrates the motions of several single lipid tracer molecules within the GUV membrane. The mobility of the tracer molecules within the model bilayers was analyzed by quantifying the single-molecule mean square displacements (MSD) as a function of time according to eq 1. A linear fit to the MSD values yielded the average diffusion coefficients of the tracer molecules in the respective GUV membrane (see Figure 2A). As expected, the 
Table 2: TR-DHPE Mobility within GUVs

\begin{tabular}{lccccc}
\hline sample & $\begin{array}{c}D_{\mathrm{MSD}}{ }^{a} \\
\left(\mu \mathrm{m}^{2} / \mathrm{s}\right)\end{array}$ & $\begin{array}{c}D_{1, \mathrm{SPT}}{ }^{b} \\
\left(\mu \mathrm{m}^{2} / \mathrm{s}\right)\end{array}$ & fraction $1^{c}$ & $\begin{array}{l}D_{2, \mathrm{SPT}}{ }^{b} \\
\left(\mu \mathrm{m}^{2} / \mathrm{s}\right)\end{array}$ & fraction $2^{c}$ \\
\hline $\mathrm{S}_{\mathrm{N}}$ & 0.6 & 0.66 & 1 & - & - \\
$\mathrm{M}_{\mathrm{N}}$ & 1.0 & 0.7 & 0.82 & 2.55 & 0.18 \\
$\mathrm{U}_{\mathrm{N}}$ & 2.7 & 2.53 & 1 & - & - \\
$\mathrm{S}_{\mathrm{A}}$ & 0.49 & 0.55 & 1 & - & - \\
$\mathrm{M}_{\mathrm{A}}$ & 1.43 & 0.87 & 0.64 & 2.48 & 0.36 \\
$\mathrm{U}_{\mathrm{A}}$ & 2.9 & 2.54 & 1 & - & - \\
\hline \multicolumn{2}{c}{ Error $\leq \pm 0.05 \mu \mathrm{m}^{2} / \mathrm{s}^{b}{ }^{b}$ Error $= \pm 0.05 \mu \mathrm{m}^{2} / \mathrm{s}}$. & ${ }^{c}$ Error $= \pm 0.05$
\end{tabular}

lipid analogues diffused faster within GUVs known to have a liquid-disordered phase $\left(D_{\mathrm{LD}}=2.7 \pm 0.05 \mu \mathrm{m}^{2} / \mathrm{s}\right)$ compared to GUVs known to comprise a liquid-ordered phase $\left(D_{\mathrm{LO}}=0.6 \pm\right.$ $0.02 \mu \mathrm{m}^{2} / \mathrm{s}$ ). The diffusion of TR-DHPE in the $\mathrm{M}_{\mathrm{N}}$ GUV s yielded an intermediate $D$ value of $1.0 \pm 0.03 \mu \mathrm{m}^{2} / \mathrm{s}$. All results are summarized in Table 2. However, confocal analysis of GUVs with mixed lipids suggested a phase separation of the ordered and unordered domains (see OSM Figure 1A of the Supporting Information for an $\mathrm{M}_{\mathrm{A}} \mathrm{GUV}$ ). In an MSD analysis, molecules with different mobility cannot be differentiated, but this can be achieved by a so-called jump distance analysis (46). In this analysis, the jumps performed by a molecule from frame to frame are evaluated. The distribution of jump distances follows a "stretched" Gaussian distribution according to eq 2 for a single diffusive species. Panels B and D of Figure 2 show the jump distance distributions for the neutral liquid-ordered and -disordered single-component GUVs, respectively. The distributions could be described well by eq 2 and yielded diffusion coefficients comparable to those from the MSD analysis (see Table 2). However, for the jump distance distribution of the neutral $\mathrm{M}_{\mathrm{N}}$ GUV, a fit according to eq 2 did not match the data. If differently mobile molecules or molecules with different mobility in domains with different viscosities exist, more than one expression according to eq 2 must be evaluated. In the case of the $\mathrm{M}_{\mathrm{N}}$ GUV, we found an excellent description of the data with two diffusion terms according to eq 3 (Figure 2C). As suggested by the confocal analysis of the mixed GUVs, we concluded that TR-DHPE is moving in two different spatially separate domains exhibiting different viscosities corresponding to two diffusion coefficients: $D_{1}=0.7 \pm 0.02 \mu \mathrm{m}^{2} / \mathrm{s}$, and $D_{2}=2.55 \pm 0.7 \mu \mathrm{m}^{2} / \mathrm{s}$. Since $\sim 80 \%$ of the jumps were observed in the more viscous liquid-ordered and only $\sim 20 \%$ were observed in the more liquid-disordered domain, we can conclude that TR-DHPE localizes preferentially in the liquid-ordered lipid domain (40). By comparing the two diffusion constants determined in the $\mathrm{M}_{\mathrm{N}}$ GUVs to the results of the single-component GUVs, we can conclude that we observed tracer diffusion in the two spatially separated liquid-ordered and liquid-disordered lipid phases. This was in good agreement with the results of ref 39, where micrometer-scale coexisting liquid phases in GUVs of the employed composition were detected. Unfortunately, it was not possible to visualize the domain structure microscopically in the GUVs used below for single-molecule imaging of TAT peptides, because of a significant spectral overlap of the available dyes for marking the domains and the spectrum of the employed fluorescent tracer molecule.

Following the analysis of lipid tracer mobility in neutral GUVs, comparable measurements were performed for anionic GUVs. We imaged TR-DHPE within anionic liquid-ordered $\left(\mathrm{S}_{\mathrm{A}}\right)$
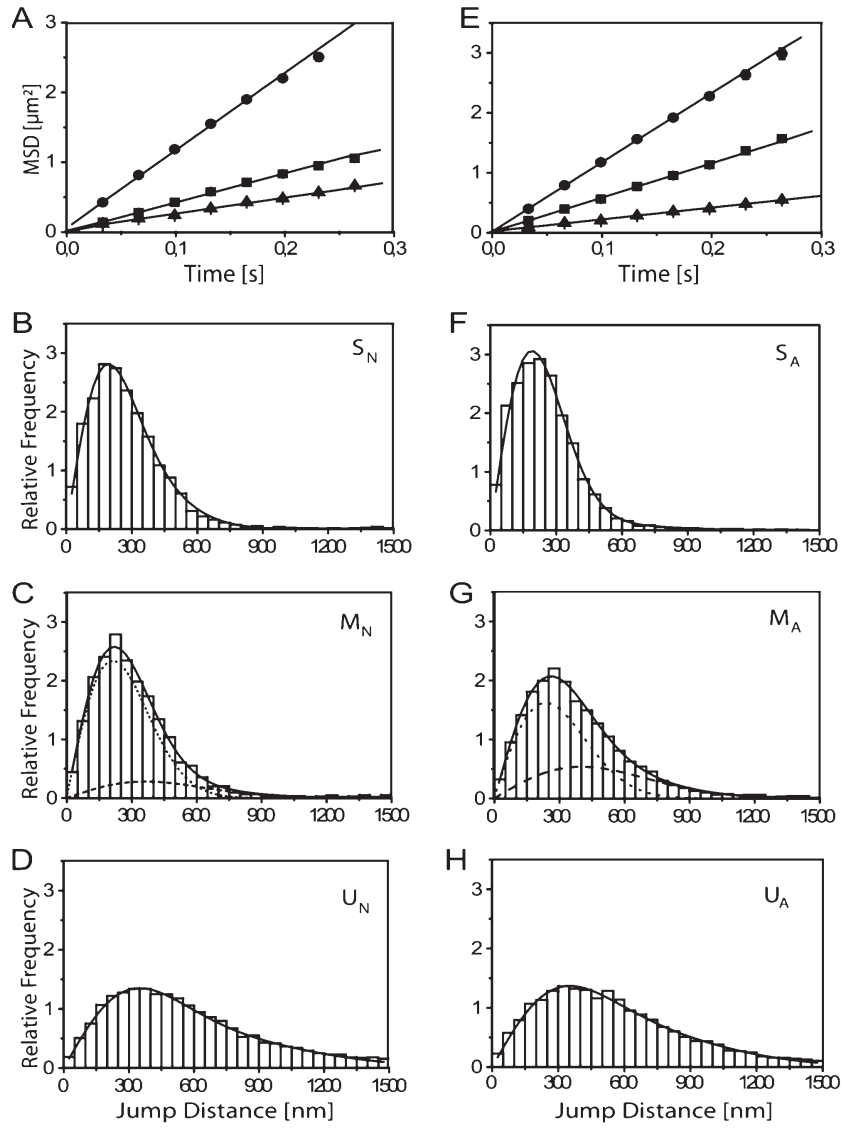

FIGURE 2: Analysis of TR-DHPE diffusion on neutral and anionic GUVs. (A) MSDs of TR-DHPE molecules plotted as a function of time for neutral membranes: data from GUVs created with $(\mathbf{\Lambda})$ DPPC and cholesterol $\left(\mathrm{S}_{\mathrm{N}}\right),(\boldsymbol{\square})$ DPPC, DOPC, and cholesterol $\left(\mathrm{M}_{\mathrm{N}}\right)$, and (๑) DOPC and cholesterol $\left(\mathrm{U}_{\mathrm{N}}\right)$. (B-D) Jump distance analysis for TR-DHPE in neutral GUVs. TR-DHPE moves more rapidly within GUVs known to have an unordered phase with a diffusion coefficient (D) of $2.53 \pm 0.05 \mu \mathrm{m}^{2} / \mathrm{s}$ compared to GUVs known to comprise an ordered phase $\left(D=0.6 \pm 0.02 \mu \mathrm{m}^{2} / \mathrm{s}\right)$. In the neutral two-component GUV, the lipid tracer showed two diffusion components, probably due to diffusion in the two different phases. (E) MSDs of TR-DHPE plotted as a function of time for anionic membranes: data for GUVs generated from (ム) DPPC, cholesterol, and DPPS $\left(\mathrm{S}_{\mathrm{A}}\right),(\boldsymbol{\square}) \mathrm{DPPC}$, DOPC, cholesterol, and DPPS $\left(\mathrm{M}_{\mathrm{A}}\right)$, and $(\bullet)$ DOPC, cholesterol, and DOPS $\left(\mathrm{U}_{\mathrm{N}}\right)$. $(\mathrm{F}-\mathrm{H})$ Jump distance analysis for TR-DHPE in anionic GUVs. Here, TR-DHPE diffuses faster within GUVs with an unordered phase with a diffusion coefficient $(D)$ of $2.54 \pm$ $0.05 \mu \mathrm{m}^{2} / \mathrm{s}$ compared to GUVs, which exhibit an ordered phase $\left(D=0.55 \pm 0.02 \mu \mathrm{m}^{2} / \mathrm{s}\right)$.

and liquid-disordered $\left(\mathrm{U}_{\mathrm{A}}\right)$ two-component GUVs, as well as anionic four-component GUVs $\left(\mathrm{M}_{\mathrm{A}}\right)$. We found that the presence of the charged lipid at $15 \mathrm{~mol} \%$ changed neither the phase behavior nor the lipid tracer mobility considerably (see Figure 2E-H and Table 2). The small changes that were observed were probably due to the fact that the cholesterol mole fraction was altered to a minor extent (40).

Confocal Imaging of TAT Peptide-GUV Interaction. For analyzing the general features of interaction of the TAT peptide with the model membranes, we incubated preformed GUVs with TAT peptides in a glucose solution. These samples were observed for extended periods of time by confocal laser scanning microscopy. For anionic GUVs, R-TAT fluorescence at the GUV surface strongly increased upon incubation with $0.5 \mu \mathrm{M}$ rhodamine-TAT (R-TAT) within $30 \mathrm{~min}$. Already after $15 \mathrm{~min}$, a distinct labeling of anionic GUVs by R-TAT could be 


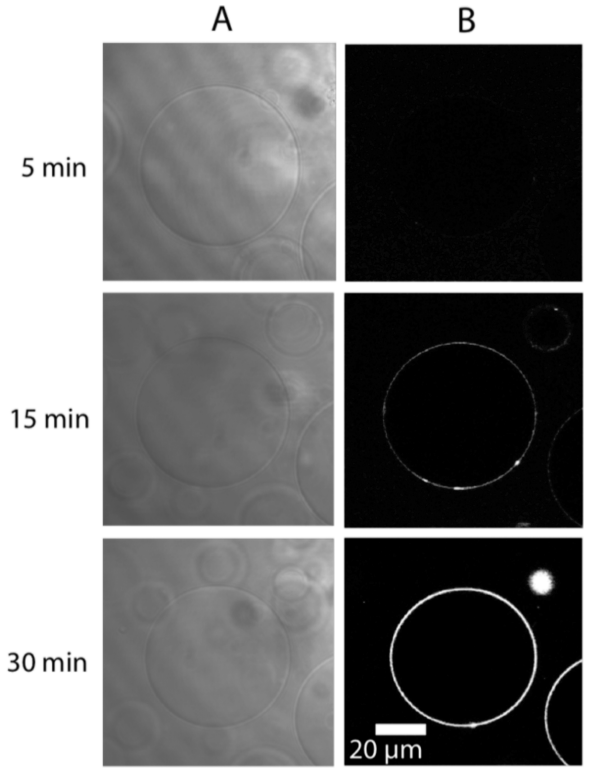

FIGURE 3: Interaction of the TAT peptide with GUVs in glucose. (A) Time series of DIC images showing the $\mathrm{M}_{\mathrm{A}}$ GUVs in a glucose solution, which were imaged in fluorescence mode for panel $\mathrm{B}$. (B) Confocal fluorescence images of the GUVs incubated with $1 \mu \mathrm{M}$ R-TAT shown in panel A in the rhodamine channel. R-TAT does not translocate the $\mathrm{M}_{\mathrm{A}}$ GUVs but just accumulates on the GUV membranes. Similar results were obtained for AF-TAT and GUVs made from all lipid mixtures used in this study.

recognized. Figure 3 shows images of exemplary $\mathrm{M}_{\mathrm{A}}$ GUVs at time points of 5, 15, and $30 \mathrm{~min}$. To simplify the detection of the GUVs, Figure 3A displays images obtained with DIC. Figure 3B shows the sample in the rhodamine fluorescence channel. A surface labeling due to TAT binding could also be observed for neutral GUVs, although the fluorescence intensity was clearly weaker (data not shown). When the R-TAT concentration was increased to $1 \mu \mathrm{M}$, a very distinct labeling of the GUVs was already clearly visible after 9 min for anionic and neutral GUVs. Again, the labeling of the anionic GUVs was stronger than that of the neutral GUVs. In a final experiment, anionic GUVs were incubated with $2 \mu \mathrm{M}$ TAT, a concentration that was well above the threshold found for efficient import of TAT into living cells (10). The GUVs were already strongly fluorescent after 2 min. However, we did not detect any R-TAT fluorescence within neutral or anionic GUVs. Obviously, the peptides were not able to translocate across the membrane at concentrations of up to $2 \mu \mathrm{M}$ under the given experimental conditions.

Lateral Mobility of Single TAT Peptides on the GUV Surface at Low Concentrations. To study the dynamics of TAT peptides on the GUV surface, we incubated neutral and anionic GUVs with $0.25 \mathrm{nM}$ R-TAT. Single-molecule imaging was started after incubation for $30 \mathrm{~min}$. The concentration of the R-TAT was so low that single diffraction-limited spots of R-TAT fluorescence became visible at the GUV surface, which could well be tracked, resulting in trajectories of TAT peptides on the GUV surface (movie S2 of the Supporting Information and Figure 4). The small fluorescent spots exhibited a higher mobility than the lipid tracers, so the imaging rate was increased from 30 to $98 \mathrm{~Hz}$ to catch the fast motions without blurring the single-molecule signals.

For the analysis of TAT peptide mobility on the GUV surface based on single-particle tracking, out-of-focus signals were sorted out using a size threshold, which was set to restrict
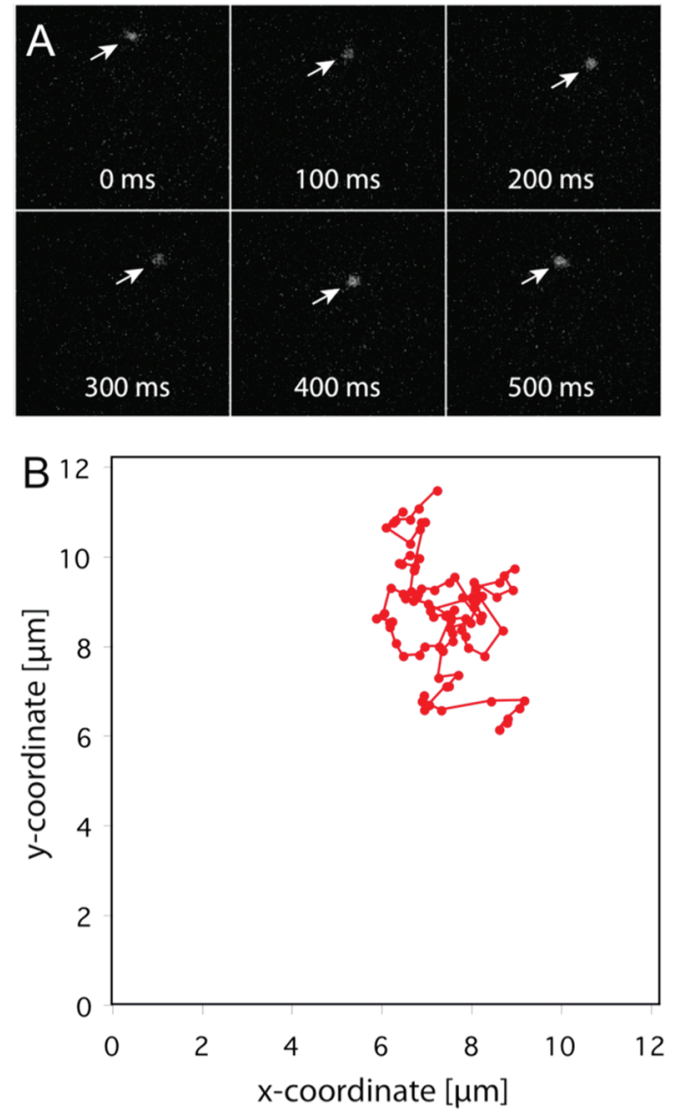

FIgURE 4: Single R-TAT peptides on the GUV surface (A) Sequence of six successive movie frames illustrating the motions of R-TAT over a time span of $0.51 \mathrm{~s}$ on anionic GUVs (DOPC, cholesterol, and DOPS at a molar ratio of 0.425:0.425:0.15). The imaging frame rate was $98 \mathrm{~Hz}$, with a single-frame integration time of $10 \mathrm{~ms}$. Only every 10th image of the image sequence is shown. The field of view of a single frame was $(12.2 \mu \mathrm{m})^{2}$. (B) Plot of the corresponding complete two-dimensional diffusion trajectory of the R-TAT molecule.

the analysis to diffraction-limited signals only corresponding to single R-TAT peptide molecules in focus. As described above for lipid tracer mobility, a MSD and a jump distance analysis of R-TAT dynamics on the GUV surface was performed for the various lipid compositions. The results of this analysis are shown in Figure 5.

The MSD plot and also the jump distance histogram clearly demonstrated a significantly higher mobility of the R-TAT than the membrane-embedded tracer molecules in all GUV systems examined (Figure 5 and Table 3). The mobility of the R-TAT peptide on the GUV surface was virtually identical for all examined GUV compositions within the precision of the measurements, and all jump distance histograms could well be fitted by a single diffusion component. This result corresponded to the LSM visualization of vesicle-attached R-TAT, which was uniform also on GUVs made from $\mathrm{M}_{\mathrm{A}}$ and $\mathrm{M}_{\mathrm{N}}$ mixtures of components (e.g., see OSM Figure 1B of the Supporting Information). Obviously, TAT peptide mobility was virtually independent of the lipid bilayer structure. A distinct difference, however, was observed for the dynamics on neutral versus anionic GUVs: TAT mobility on neutral GUV surfaces was higher than on anionic GUVs as shown by the average diffusion coefficient on neutral GUVs $\left(D_{\mathrm{N}, \mathrm{SPT}}=6.2 \pm 0.6 \mu \mathrm{m}^{2} / \mathrm{s}\right)$, whereas it amounted on anionic GUVs only to a $D_{\mathrm{A}, \mathrm{SPT}}$ of $3.5 \pm 0.6 \mu \mathrm{m}^{2} / \mathrm{s}$. 

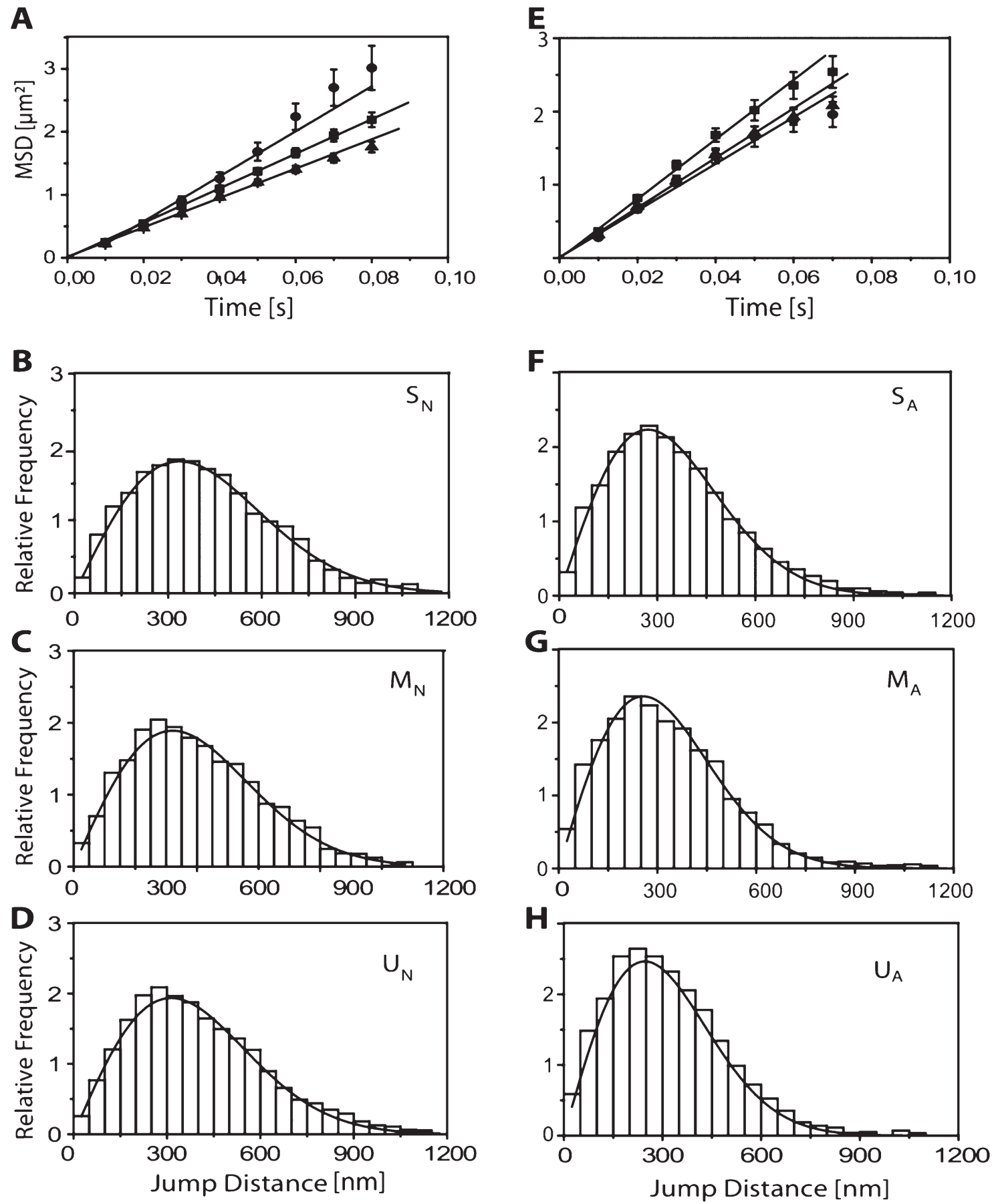

FIgURE 5: Analysis of R-TAT diffusion on neutral and anionic GUVs at a low concentration (0.25 nM). (A) Plot of MSD vs time for R-TAT on

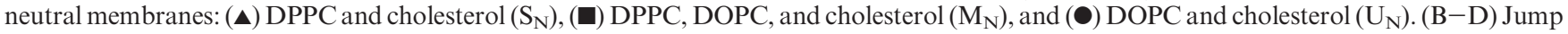
distance analysis for R-TAT in neutral GUVs for the different GUV compositions as indicated in the plots. (E) Plot of MSD vs time for of R-TAT on anionic membranes: (ム) DPPC, cholesterol, and DPPS $\left(\mathrm{S}_{\mathrm{A}}\right),(\boldsymbol{\square})$ DPPC, DOPC, cholesterol, and DPPS (M $)_{\mathrm{A}}$ ), and $(\bullet)$ DOPC, cholesterol, and DOPS $\left(\mathrm{U}_{\mathrm{N}}\right)$. $(\mathrm{F}-\mathrm{H})$ Jump distance analysis for R-TAT in anionic GUVs for the different GUV compositions as indicated in the plots.

The TAT peptide trajectories were considerably shorter than those of the lipid tracers in terms of the average number of frames, in which single molecules were observed sequentially. This indicated a rapid exchange of TAT peptides between solution and the surface of neutral and anionic GUVs. Also, in experiments with neutral GUVs, the TAT trajectories were shorter then those observed with anionic GUVs. We assume that this was due to an increased strength of binding of the cationic TAT to the anionic GUVs in comparison of the neutral ones, which was already observed in the confocal microscope. The duration of binding of the
R-TAT molecules to the GUVs was quantified by evaluating the distribution of the trajectory lengths. These are plotted in histogram form in Figure 6. The track length distributions showed a rapid decrease, which was approximately exponential. By fitting monoexponential decay functions to these binding time distributions, we determined in the case of the neutral GUVs an average interaction duration of $13 \mathrm{~ms}$, whereas it amounted to $20 \mathrm{~ms}$ in the case of the anionic GUVs. Short trajectories are problematic for the MSD analysis, because only relatively few data points contribute to the calculation of the MSD at longer times, causing strong 
data fluctuation. Therefore, we used only the first five time points to determine the diffusion constant corresponding to times up to $50 \mathrm{~ms}$.

Lateral Mobility of Single TATs on the GUV Surface at High Concentrations. The optical analysis of R-TAT binding to GUVs revealed that at a concentration of at least $0.5 \mu \mathrm{M}$ TAT peptides efficiently bind to and accumulate on the vesicle surface, especially in the case of anionic GUVs. To study the dynamics of TAT peptides within such a "peptide layer", we incubated the GUVs with up to $2 \mu \mathrm{M}$ nonfluorescent b-TAT supplemented with traces of R-TAT $(0.1 \mathrm{nM})$. This guaranteed that even in the presence of a high overall TAT concentration single fluorescent TAT peptides would still be sufficiently sparse to be discerned on the GUV surface. Single-molecule imaging was started after incubation for 30 min. As described above, we acquired movies revealing single-TAT peptide mobility on the GUV surface and analyzed their mobility. We found the mobility of R-TAT peptides on the GUV surface to be almost identical for all examined GUV compositions within the precision of the measurements, and all jump distance histograms could well be fitted by a single diffusion component. The jump distance analysis revealed no reduction in the mobility of R-TAT in comparison to that in the low-concentration situation (see Table 3).

\section{DISCUSSION.}

Previous detailed biophysical characterization of the structure and interactions of PTDs within membranes allowed important insights into the energetics of peptide translocation and the mechanisms of penetration

Table 3: R-TAT Peptide Mobility on GUVs

\begin{tabular}{|c|c|c|c|c|}
\hline \multirow[b]{2}{*}{ sample } & \multicolumn{2}{|c|}{$0.25 \mathrm{nM}$ R-TAT } & \multicolumn{2}{|c|}{$0.1 \mathrm{nM}$ R-TAT and $2 \mu \mathrm{M}$ b-TAT } \\
\hline & $D_{\mathrm{MSD}}{ }^{a}\left(\mu \mathrm{m}^{2} / \mathrm{s}\right)$ & $D_{\mathrm{SPT}}^{b}\left(\mu \mathrm{m}^{2} / \mathrm{s}\right)$ & $D_{\mathrm{MSD}}{ }^{a}\left(\mu \mathrm{m}^{2} / \mathrm{s}\right)$ & $D_{\mathrm{SPT}}^{b}\left(\mu \mathrm{m}^{2} / \mathrm{s}\right)$ \\
\hline $\mathrm{S}_{\mathrm{N}}$ & 6.8 & 5.7 & 5.5 & 5.3 \\
\hline $\mathrm{M}_{\mathrm{N}}$ & 5.6 & 4.9 & 5.6 & 4.8 \\
\hline $\mathrm{U}_{\mathrm{N}}$ & 6.3 & 5.0 & 5.1 & 4.9 \\
\hline $\mathrm{S}_{\mathrm{A}}$ & 3.6 & 3.7 & 4.0 & 3.4 \\
\hline $\mathrm{M}_{\mathrm{A}}$ & 4.0 & 3.3 & 4.2 & 3.3 \\
\hline $\mathrm{U}_{\mathrm{A}}$ & 3.3 & 3.0 & 3.9 & 3.2 \\
\hline
\end{tabular}

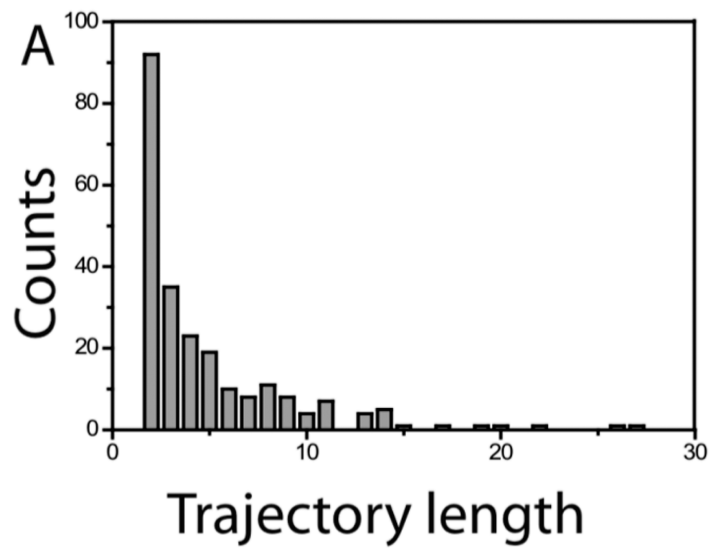

processes $(20,30,31)$. An especially well-studied PTD is the TAT peptide, and its cell penetrating capabilities have been characterized in detail (10). Tunnemann et al. demonstrated by in vivo translocation studies that fluorescently TAT peptides efficiently translocate into living cells.

Numerous studies exist on the structures, which are adopted by PTDs in solution and in membranes, but not much is known about their mobility on or within membranes. We studied for the first time the dynamics of the TAT PTD on well-defined membrane model systems using single-molecule microscopy, which is particularly well suited to discerning possibly existing mobility fractions. To begin, we analyzed the mobility of a lipid tracer molecule, TR-DHPE, within GUV membranes made from lipid mixtures known to form liquid-ordered membranes, liquiddisordered membranes, and membrane domains. This part of the study provided us with reference mobility values for lipids within the employed model membranes. We found that the mobility of TR-DHPE varied from 0.5 to $2.5 \mu \mathrm{m}^{2} / \mathrm{s}$ for liquid-ordered and liquid-disordered membranes for the various lipid compositions examined. Furthermore, we found that addition of $15 \mathrm{~mol} \%$ anionic lipids did not change the tracer mobility considerably. As in approaches using FCS, we detected two different diffusion coefficients when systems of lipids were employed, which are known to exhibit micrometer-sized domains of different composition and mobility (39).

We observed a strong and fast labeling of the GUV surface by TAT peptides, which was more efficient for anionic GUVs compared to neutral GUVs. The binding did not exhibit any lipid domain correspondence. GUVs were labeled strongly within minutes, but no TAT was detected within the GUV interior.

The mobility of TAT peptides bound on the vesicle surface was significantly higher than that of the membrane-immersed tracer molecules and not dependent on the phase state of the model bilayer. In contrast to the lipid mobility, the diffusion of the cationic TAT peptides was clearly slowed in membranes containing $15 \mathrm{~mol} \mathrm{\%}$ anionic lipids. Taken together, these observations suggested that the TAT peptides were not immersed within the viscous hydrophobic domain of the membrane but were rather "floating" on the headgroup domain of the bilayer. In the presence of anionic lipids in the membrane, the cationic TAT peptides are attracted by the negatively charged lipid headgroups causing prolonged attachments and a reduced mobility of peptides in comparison to neutral membranes. The latter

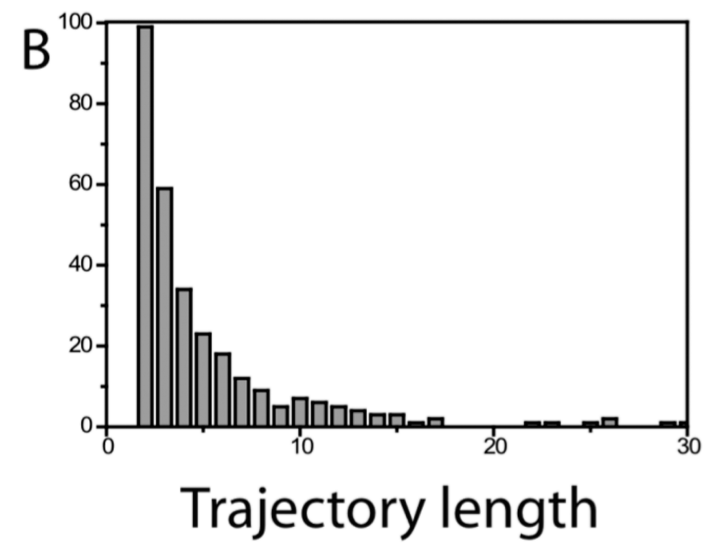

FIGURE 6: Distribution of the trajectory lengths Histograms quantifying the trajectory length of R-TAT molecules on neutral (A) and (B) anionic GUVs. The duration of binding of the R-TAT molecules to neutral GUVs was shorter compared to that with anionic GUVs. Fits by a monoexponential decay function indicated binding times of 13 and $20 \mathrm{~ms}$ for neutral and anionic GUVs, respectively. 
observation suggested a deeper incorporation of the cationic peptide into the anionic lipid headgroup region. Quantitative studies of TAT-mediated cell translocation of cargo molecules suggested the existence of a limiting concentration, at which effective penetration would occur (10). We also detected a strong concentration-dependent tendency of the TAT peptides to accumulate on the model bilayers. Therefore, we studied the peptide mobility also at a concentration at which extensive TAT - membrane association could be observed up to a concentration that would lead in cells to peptide uptake $(\sim 2 \mu \mathrm{M})$. Such a situation might correspond to the situation suggested in the carpet model of peptide translocation (47). However, we found no significant change in TAT mobility even at TAT concentrations, which yielded an efficient membrane staining within minutes. This suggested that even under these conditions the TAT peptides are still relatively diluted on the membrane and do not form pore complexes or carpetlike structures. No glycosaminoglycans were present in the GUV membranes, which were suggested to play a role in mediating interactions between TAT and cell membranes (21, 22), but still a strong binding, probably due to charge interaction, was evident. An extension of the studies presented in this paper to antimicrobial peptides, which supposedly act by membrane pore formation, will be most instructive.

\section{ACKNOWLEDGMENT}

M.C.C. and G.T. thank Andreas Herrmann (Humboldt University, Berlin, Germany) for his help in the initial GUV experiments, and U.K. and C.C. thank Jan Peter Siebrasse for stimulating discussions on this work.

\section{SUPPORTING INFORMATION AVAILABLE}

A figure depicting confocal imaging of GUVs, movie 1 which documents single-molecule imaging of TR-DHPE in a twocomponent GUV, and movie 2 which documents single-molecule imaging of R-TAT peptides in an anionic two-component GUV. This material is available free of charge via the Internet at http://pubs.acs.org.

\section{REFERENCES}

1. Dietz, G. P., and Bahr, B. (2004) Delivery of bioactive molecules into the cell: The Trojan horse approach. Mol. Cell. Neurosci. 27, 85-131.

2. Richard, J. P., Melikov, K., Vives, E., Ramos, C., Verbeure, B., Gait, M. J., Chernomordik, L. V., and Lebleu, B. (2003) Cell-penetrating peptides. A reevaluation of the mechanism of cellular uptake. J. Biol. Chem. 278, 585-590.

3. Schwarze, S. R., Hruska, K. A., and Dowdy, S. F. (2000) Protein transduction: Unrestricted delivery into all cells?. Trends Cell Biol. 10, 290-295.

4. Lindgren, M., Hallbrink, M., Prochiantz, A., and Langel, U. (2000) Cell-penetrating peptides. Trends Pharmacol. Sci. 21, 99-103.

5. Derossi, D., Chassaing, G., and Prochiantz, A. (1998) Trojan peptides: The penetratin system for intracellular delivery. Trends Cell Biol. 8, 84-87.

6. Kaplan, I. M., Wadia, J. S., and Dowdy, S. F. (2005) Cationic TAT peptide transduction domain enters cells by macropinocytosis. J. Controlled Release 102, 247-253.

7. Fretz, M. M., Penning, N. A., Al-Taei, S., Futaki, S., Takeuchi, T., Nakase, I., Storm, G., and Jones, A. T. (2007) Temperature-, concentration- and cholesterol-dependent translocation of L- and D-octa-arginine across the plasma and nuclear membrane of CD34 + leukaemia cells. Biochem. J. 403, 335-342.

8. Duchardt, F., Fotin-Mleczek, M., Schwarz, H., Fischer, R., and Brock, R. (2007) A comprehensive model for the cellular uptake of cationic cell-penetrating peptides. Traffic 8, 848-866.

9. Ter-Avetisyan, G., Tunnemann, G., Nowak, D., Nitschke, M., Herrmann, A., Drab, M., and Cardoso, M. C. (2008) Cell entry of arginine-rich peptides is independent of endocytosis. J. Biol. Chem. 284, 3370-3378.

10. Tunnemann, G., Martin, R. M., Haupt, S., Patsch, C., Edenhofer, F., and Cardoso, M. C. (2006) Cargo-dependent mode of uptake and bioavailability of TAT-containing proteins and peptides in living cells. FASEB J. 20, 1775-1784.

11. Fischer, R., Fotin-Mleczek, M., Hufnagel, H., and Brock, R. (2005) Break on through to the other side: Biophysics and cell biology shed light on cell-penetrating peptides. ChemBioChem 6, 21262142 .

12. Torchilin, V. P. (2006) Recent approaches to intracellular delivery of drugs and DNA and organelle targeting. Annu. Rev. Biomed. Eng. 8, 343-375.

13. Rothbard, J. B., Kreider, E., VanDeusen, C. L., Wright, L., Wylie, B. L., and Wender, P. A. (2002) Arginine-rich molecular transporters for drug delivery: Role of backbone spacing in cellular uptake. J. Med. Chem. 45, 3612-3618.

14. Tunnemann, G., Ter-Avetisyan, G., Martin, R. M., Stockl, M., Herrmann, A., and Cardoso, M. C. (2008) Live-cell analysis of cell penetration ability and toxicity of oligo-arginines. J. Pept. Sci. 14, 469-476.

15. Drin, G., Cottin, S., Blanc, E., Rees, E. R., and Temsamani, J. (2003) Studies on the internalization mechanism of cationic cell-penetrating peptides. J. Biol. Chem. 278, 31192-31201.

16. Ferrari, A., Pellegrini, V., Arcangeli, C., Fittipaldi, A., Giacca, M., and Beltram, F. (2003) Caveolae-mediated internalization of extracellular HIV-1 tat fusion proteins visualized in real time. Mol. Ther. 8, 284-294.

17. Khalil, I. A., Kogure, K., Futaki, S., and Harashima, H. (2006) High density of octaarginine stimulates macropinocytosis leading to efficient intracellular trafficking for gene expression. J. Biol. Chem. 281, 3544-3551.

18. Thoren, P. E., Persson, D., Esbjorner, E. K., Goksor, M., Lincoln, P., and Norden, B. (2004) Membrane binding and translocation of cellpenetrating peptides. Biochemistry 43, 3471-3489.

19. Shai, Y. (2002) Mode of action of membrane active antimicrobial peptides. Biopolymers 66, 236-248.

20. Patel, L. N., Zaro, J. L., and Shen, W. C. (2007) Cell penetrating peptides: Intracellular pathways and pharmaceutical perspectives. Pharm. Res. 24, 1977-1992.

21. Ziegler, A., and Seelig, J. (2004) Interaction of the protein transduction domain of HIV-1 TAT with heparan sulfate: Binding mechanism and thermodynamic parameters. Biophys. J. 86, 254-263.

22. Ziegler, A., and Seelig, J. (2008) Binding and clustering of glycosaminoglycans: A common property of mono- and multivalent cell-penetrating compounds. Biophys. J. 94, 2142-2149.

23. Frankel, A. D., and Pabo, C. O. (1988) Cellular uptake of the tat protein from human immunodeficiency virus. Cell 55, 11891193.

24. Tyagi, M., Rusnati, M., Presta, M., and Giacca, M. (2001) Internalization of HIV-1 tat requires cell surface heparan sulfate proteoglycans. J. Biol. Chem. 276, 3254-3261.

25. Rothbard, J. B., Jessop, T. C., and Wender, P. A. (2005) Adaptive translocation: The role of hydrogen bonding and membrane potential in the uptake of guanidinium-rich transporters into cells. Adv. Drug Delivery Rev. 57, 495-504.

26. Prochiantz, A. (2000) Messenger proteins: Homeoproteins, TAT and others. Curr. Opin. Cell Biol. 12, 400-406.

27. Vogel, B. E., Lee, S. J., Hildebrand, A., Craig, W., Pierschbacher, M. D., Wong-Staal, F., and Ruoslahti, E. (1993) A novel integrin specificity exemplified by binding of the $\alpha \mathrm{v} \beta 5$ integrin to the basic domain of the HIV Tat protein and vitronectin. J. Cell Biol. 121, 461-468.

28. Weeks, B. S., Desai, K., Loewenstein, P. M., Klotman, M. E., Klotman, P. E., Green, M., and Kleinman, H. K. (1993) Identification of a novel cell attachment domain in the HIV-1 Tat protein and its 90-kDa cell surface binding protein. J. Biol. Chem. 268, 52795284.

29. Derossi, D., Calvet, S., Trembleau, A., Brunissen, A., Chassaing, G., and Prochiantz, A. (1996) Cell internalization of the third helix of the Antennapedia homeodomain is receptor-independent. J. Biol. Chem. 271, 18188-18193.

30. Tiriveedhi, V., and Butko, P. (2007) A fluorescence spectroscopy study on the interactions of the TAT-PTD peptide with model lipid membranes. Biochemistry 46, 3888-3895.

31. Ziegler, A., Blatter, X. L., Seelig, A., and Seelig, J. (2003) Protein transduction domains of HIV-1 and SIV TAT interact with charged lipid vesicles. Binding mechanism and thermodynamic analysis. Biochemistry 42, 9185-9194. 
32. Siebrasse, J. P., Grunwald, D., and Kubitscheck, U. (2007) Singlemolecule tracking in eukaryotic cell nuclei. Anal. Bioanal. Chem. 387, 41-44.

33. Thompson, R. E., Larson, D. R., and Webb, W. W. (2002) Precise nanometer localization analysis for individual fluorescent probes. Biophys. J. 82, 2775-2783.

34. Saxton, M. J., and Jacobson, K. (1997) Single-particle tracking: Applications to membrane dynamics. Annu. Rev. Biophys. Biomol. Struct. 26, 373-399.

35. Schütz, G. J., Sonnleitner, M., Hinterdorfer, P., and Schindler, H. (2000) Single molecule microscopy of biomembranes. Mol. Membr. Biol. 17, 17-29

36. Sako, Y., and Yanagida, T. (2003) Single-molecule visualization in cell biology. Nat. Cell Biol. 5(Suppl), Ss1-Ss5.

37. Dimitrov, D. S., and Angelova, M. I. (1988) Lipid swelling and liposome formation mediated by electric fields. Bioelectrochem. Bioenerg. 19, 323-336.

38. Menger, F. M., and Angelova, M. I. (1998) Giant Vesicles: Imitating the Cytological Process of Cell Membranes. Acc. Chem. Res. 31, 789-797.

39. Veatch, S. L., and Keller, S. L. (2003) Separation of liquid phases in giant vesicles of ternary mixtures of phospholipids and cholesterol. Biophys. J. 85, 3074-3083.
40. Scherfeld, D., Kahya, N., and Schwille, P. (2003) Lipid dynamics and domain formation in model membranes composed of ternary mixtures of unsaturated and saturated phosphatidylcholines and cholesterol. Biophys. J. 85, 3758-3768.

41. Abramoff, M. D., Magelhaes, P. J., and Ram, S. J. (2004) Image Processing with ImageJ. Biophot. Int. 11, 36-42.

42. Crank, J. (1975) The Mathematics of Diffusion, Clarendon Press, Oxford, U.K.

43. Schmidt, T., Schütz, G. J., Baumgartner, W., Gruber, H. J., and Schindler, H. (1995) Characterization of photophysics and mobility of single molecules in a fluid lipid membrane. J. Chem. Phys. 99, 17662-17668.

44. Murcia, M. J., Garg, S., and Naumann, C. A. (2007) Single-molecule fluorescence microscopy to determine phospholipid lateral diffusion. Methods Mol. Biol. 400, 277-294.

45. Alberts, B., Johnson, A., Lewis, J., Raff, M., Roberts, K., and Walter, P. (2008) Molecular Biology of the Cell, Taylor \& Francis, New York.

46. Kues, T., Peters, R., and Kubitscheck, U. (2001) Visualization and tracking of single protein molecules in the cell nucleus. Biophys. J. 80, 2954-2967.

47. Shai, Y. (1999) Mechanism of the binding, insertion and destabilization of phospholipid bilayer membranes by $\alpha$-helical antimicrobial and cell non-selective membrane-lytic peptides. Biochim. Biophys. Acta 1462, 55-70. 


\section{Online supplemental material:}

\section{OSM Fig 1: Confocal imaging of GUVs}

(A) Extended focus images of confocal image stacks of lipid domains in $M_{A}$ GUVs labeled with BodipyPC, which distributes into liquid-disordered lipid domains containing mostly unsaturated DOPC. (B) In contrast to labeling by BodipyPC incubation of $M_{A}$ GUVs with RTAT yields a uniform distribution.
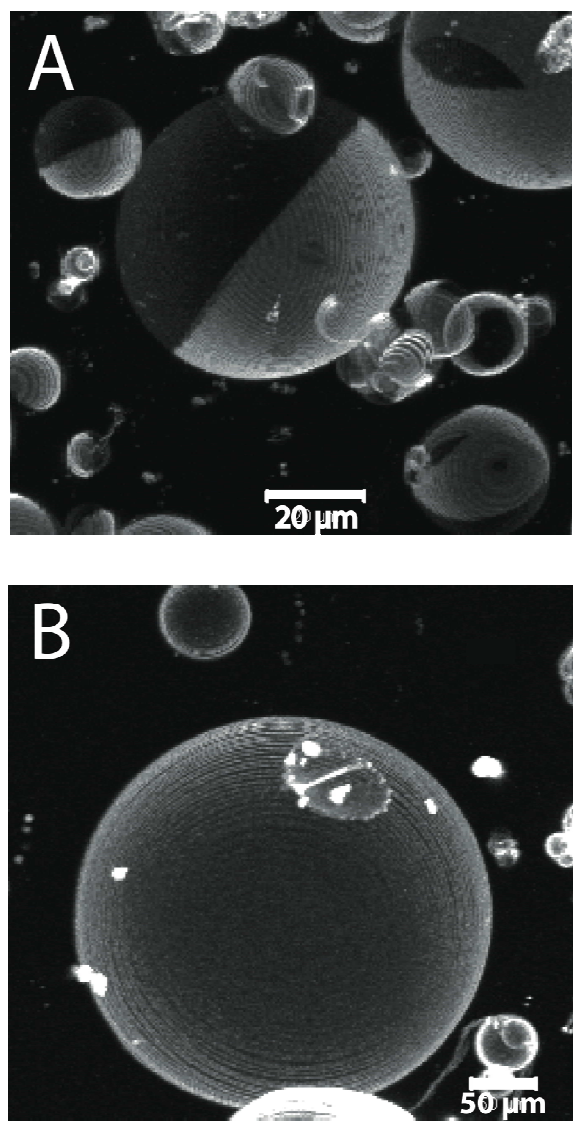

OSM Fig. 1 Ciobanasu et al. 


\section{Supplemental Movie S1:}

Single molecule imaging of TR-DHPE in a two-component GUV

We imaged the tracer TR-DHPE within a neutral two-component GUV

(DOPC:DPPC:cholesterol was 1:1:1). The tracer added to the lipids at a ratio of $10^{-7} \mathrm{~mol} \%$. Single diffraction-limited fluorescence spots representing single TR-DHPE molecules could be observed diffusing within the GUV membrane in the high-speed video microscope operated at a frame rate of $30 \mathrm{~Hz}$. The original video data were smoothed by a $3 \times 3$ mean value kernel, and linearly contrast enhanced. The field of view was $12.2 \times 12.2 \mu \mathrm{m}^{2}$.

\section{Supplemental Movie S2:}

Single molecule imaging of R-TAT peptides in an anionic two component GUV

We imaged R-TAT peptides within a neutral two-component GUV (DOPC:DPPC:cholesterol was $1: 1: 1)$. The peptides were added at a concentration of $0.25 \mathrm{nM}$ to GUVs. Single diffraction-limited fluorescence spots representing single R-TAT peptide molecules could be observed diffusing on the GUV membrane in the high-speed video microscope operated at a frame rate of $100 \mathrm{~Hz}$. The movie is here displayed at $30 \mathrm{~Hz}$ at a 3.3-fold slow motion. The original video data were smoothed by a $3 \times 3$ mean value kernel, and linearly contrast enhanced. The field of view was $12.2 \times 12.2 \mu \mathrm{m}^{2}$. 